\title{
Polymyositis Mimicking Inflammatory Dystrophy
}

\author{
Ramnath Santosh Ramanathan, MD \\ Staff Neurology, Vascular Neurology, Akron Neurology Inc, Akron, USA
}

*Corresponding Author: Ramnath Santosh Ramanathan, Staff Neurology, Vascular Neurology, Akron Neurology Inc, Akron, US A. E-mail:dr.santosh7@gmail.com

Received: April 12, 2017

Accepted: April 15, 2017
Published: April 17, 2017

A 48 year Caucasian female presented with 6 to 8 month history of upper and lower extremity weakness, where she had more difficulty in holding objects with her hands, and had distal weakness in her hands with difficulty in doing fine motor skills. She also complained of proximal weakness of both her lower extremities and had difficulty in getting up from the sitting position. She was reported to have myotonic discharges in the electromyography and nerve conduction studies (EMG/NCS) done at outside hospital and diagnosed with possible myotonic dystrophy. On admission her vitals were normal and on neurology examination pertinent findings included distal $3 / 5$ muscle weakness in both upper extremities at wrist flexion, extension as well as in the small muscles of hand, $4 / 5$ weakness at elbow flexion and extension as well as at the shoulder. She had $4 / 5$ weakness at hip flexion and extension, abduction and adduction with $5 / 5$ muscle strength at more distal examination in the lower extremities bilaterally. She had decreased muscle stretch reflex throughout and had normal sensory examination. There were no findings on examination to suggest myotonic dystrophy. Her CPK level was greater than 1000 units and EMG/NCS showed myopathic motor unit potentials and increased spontaneous activity with fibrillations, positive sharp waves as well asmyotonic discharges. Given myopathy was higher on the differential patient underwent muscle biopsy at right deltoid muscle (figure) which showed evidence of perivascular inflammation within the endomysium without any necrotic tissue or vacuoles. Patient was treated with high dose steroids with improvement in muscle strength and plan is to treat her with long term immunosuppressive therapy.

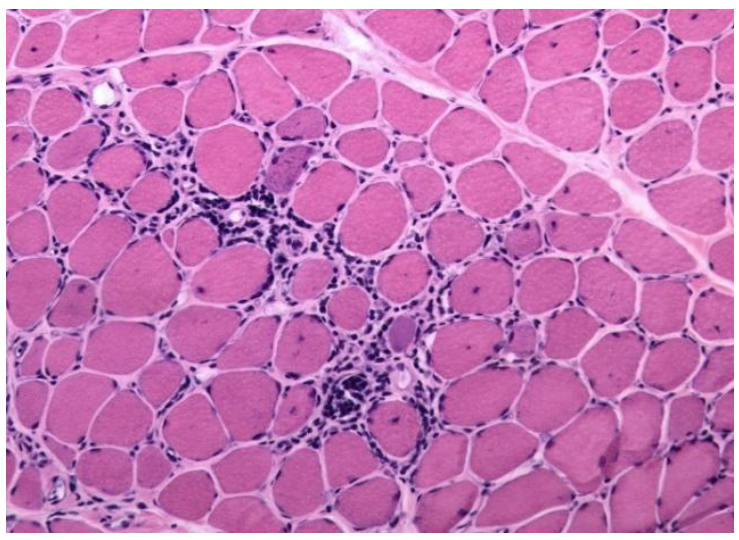

Figure1. High power resolution

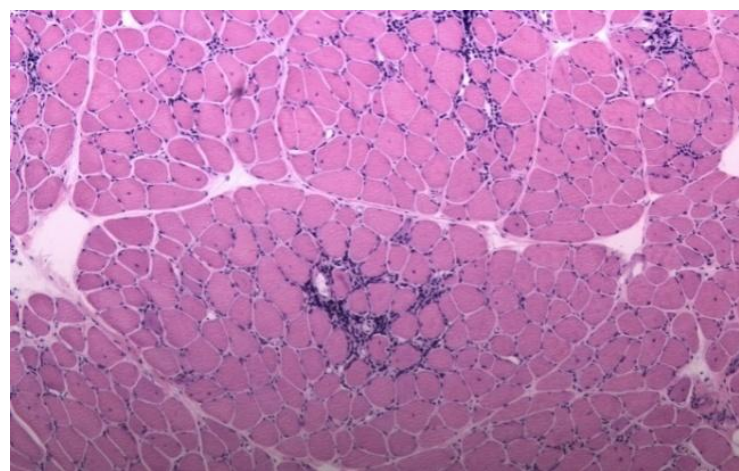

Figure2. Perivascular inflammation within the endomysium without any necrosis. Discussion

Inflammatory myopathies have been divided into four subtypes including polymyositis, dermatomyositis, necrotizing autoimmune myositis and inclusion body myositis with the fifth subtype being overlap myositis. The existence of pure polymyositis though has been challenged recently due to the new pathological classification of autoimmune myositis including necrotizing autoimmune myositis which is linked to 3-hydroxy-3-methylglutaryl coenzyme A reductase (anti-HMGCR) or anti-signal recognition particle (anti-SRP) and is seen after statin exposure. The necrotizing autoimmune myositis is viewed as an entity distinct than the pure polymyositis $(1,2)$. Polymyositis is generally a diagnosis of exclusion and has a sub 
acute clinical course associated with proximal weakness, without any involvement of facial or ocular muscles. Polymyositis can be associated with antisynthetase antibody (anti-Jo antibody) which in turn is associated with interstitial lung disease, Raynaud's phenomenon and mechanic's hands. Polymyositis is associated with endomysial inflammation and invasion by CD $8 \mathrm{~T}$ cells of the nonnecrotic muscle fibers expressing major histocompatibility expressing (MHC) class 1 antigen cells, which differentiates it from muscular dystrophies (3). Polymyositis is generally responsive to steroids, immunosuppressive agents as well as intravenous immune globulin therapy in appropriate case scenarios.

\section{REFERENCES}

[1] Dalakas MC. Inflammatory muscle diseases. N Engl J Med 2015; 372:1734-1747.

[2] Quinn C, Salameh JS, Smith T, et al. Necrotizing myopathies: an update. J Clin NeuromuscDisord2015; 16:131-40.

[3] Dalakas MC, Hohlfeld R. Polymyositis and dermatomyositis. Lancet 2003;362:971-982

Citation: Ramnath Santosh Ramanathan. Polymyositis Mimicking Inflammatory Dystrophy. ARC Journal of Neuroscience 2017; 2(1):1-2. doi:dx.doi.org/10.20431/2456-057X.0201001.

Copyright: (C) 2017 Authors. This is an open-access article distributed under the terms of the Creative Commons Attribution License, which permits unrestricted use, distribution, and reproduction in any medium, provided the original author and source are credited. 